\title{
Comparable Effects of Nicotine in Smokers and Nonsmokers on a Prospective Memory Task
}

\author{
Jennifer M Rusted*,' and Steven Trawley' \\ 'Department of Psychology, Sussex University, Brighton, Sussex, UK
}

\begin{abstract}
In a double-blind placebo-controlled study, we examined the effect of nicotine, a cholinergic agonist, on performance of a prospective memory (ProM) task in young adult volunteers. Volunteers were required to complete an ongoing lexical decision task while maintaining the ProM task (responding with a different button press to items containing particular target letters). Half of the volunteers were smokers, half were nonsmokers. Half of each group received a single dose ( I mg) of nicotine nasal spray before completing the task; the remaining volunteers received a matched inactive placebo spray. Nicotine improved performance on the ProM task when volunteers were able to devote resources to that task. Under a variant procedure, where volunteers completed a concurrent auditory monitoring task, ProM performance was impaired under nicotine. Results are discussed in terms of the resource model of ProM, and the arousal model of drug effects. The data suggest that ProM under the conditions tested here is a resource-needy process, and that nicotine can improve performance by increasing available resources. Increased working memory demands that encourage redirection of resources may impair ProM performance, but the conditions under which these deficits emerge depend upon the subjective allocation of resources across tasks, rather than resource availability per se.

Neuropsychopharmacology (2006) 3 I, I545-1549. doi: I 0. I 038/sj.npp. I 300965; published online 23 November 2005
\end{abstract}

Keywords: nicotine; prospective memory; working memory; attention; arousal

\section{INTRODUCTION}

The successful management of our everyday lives frequently requires us to form plans or intentions about future actions. Intentions that cannot be immediately performed have to be maintained until the appropriate opportunity occurs. Common activities such as shopping or posting a letter are examples of intentions that are time linked to the situations in which they can be realized. This process of forming a delayed intention is known as prospective memory (ProM) (Einstein and McDaniel, 1990; Ellis and Kvavilashvili, 2000). Laboratory-based studies of ProM (Cherry and LeCompte, 1999; Kidder et al, 1997; Kliegel et al, 2003; Logie et al, 2004; Mantyla and Nilsson, 1997) and more naturalistic studies (Maylor, 1996; Huppert and Beardsall, 1993) report agerelated decline in these skills. Critically, the efficiency of our ProM capability impacts directly on our ability to live independently.

Recently, there has been considerable research examining the nature of the processes that comprise ProM, indicating the existence of both strategic and automatic intention retrieval (Einstein and McDaniel, 1996; McDaniel and

\footnotetext{
*Correspondence: Dr JM Rusted, Department of Psychology, Sussex University, Brighton, Sussex BNI 9QG, UK, Tel: + 441273678325 , Fax: + 441273 678058, E-mail: jennyr@sussex.ac.uk

Received 3 August 2005; revised 3 October 2005; accepted 4 October 2005

Online publication: 13 October 2005 at http://www.acnp.org/citations/ Npp I01305050489/default.pdf
}

Einstein, 2000; Einstein et al, 2005; Smith and Bayen, 2004). Whether strategic or automatic processes are engaged is likely to be both task and resource dependent (McDaniel et al, 2004; Einstein et al, 2005; Kliegel et al, 2004) and may be influenced by the contextual cues available to support retrieval (Nowinski and Dismukes, 2005; Trawley and Rusted, under review). The emerging evidence for the engagement of strategic processing in some aspects of ProM retrieval are consistent with the involvement of frontal or executive function in planning and in delayed implementation of plans or intentions (eg Shallice and Burgess, 1996; Burgess et al, 2003). Indeed, there is converging evidence from neuroimaging studies that Brodmann's area 10, within the rostral frontal cortex, has special significance in the maintenance (as opposed to the execution) of an intention (Burgess et al, 2001; Okuda et al, 1998; Burgess et al, 2003).

If there are neuroanatomically specific sites activated by intention planning and maintenance, it begs the question as to the underlying neuropharmacology that supports such processes. Understanding the neurochemistry of ProM offers not only the opportunity for pharmacological interventions to support those processes in older adults but also a means of validating the psychological models of ProM, that is, whether purported processes are truly dissociable.

In the past few years, exploration of the neurochemical systems maintaining efficient information processing in human volunteers and animals has continued to emphasize 
the central role of the cholinergic system (Robbins, 2002). With regard to processes associated with working memory and strategic processing of information, the cholinergic agonist nicotine has been demonstrated to improve performance (Warburton et al, 2001; Rusted et al, 1998; Mancuso et al, 1999; Edginton and Rusted, 2003; Ernst et al, 2001; Levin et al, 1998; Kumari et al, 2003; Rycroft et al, 2005). Recently, Rusted et al (in press) reported three studies exploring nicotine's effects on ProM performance. The results were consistent with the view that when the ProM task engages strategic (ie, effortful) processes, nicotine delivered to 2 -h abstinent smokers via smoking of a single cigarette produced a significant improvement in ProM accuracy. The study examined a combination of conditions. The ProM task was either actively maintained (vigilance instruction), presented as subjugate to the lexical decision task (LDT) (prospective condition), or the volunteer worked under dual task (LDT + PM) instructions; the ProM targets were either letters within the LDT stimuli, or the LDT stimuli themselves. All of these combinations were completed either with or without nicotine. Nicotine improved ProM performance only when the ProM task required distinct processing from the ongoing task (when the ProM target were letters within the stimuli presented for a lexical decision, but not when the targets were the words themselves), or when the volunteer had been primed to the dual nature of the task requirements and was active in the selective allocation of resources between the ProM and the LDT. In the latter condition, nicotine-related improvement in ProM accuracy was independent of ProM target type and of the task prioritized. Two issues arise from this study, namely, how is nicotine influencing ProM accuracy and can the effects be observed in nonsmokers?

Regarding the mechanism of action, if nicotine increases the cognitive resource that an individual brings to the task, then one might expect an interaction between load and nicotine, since an additional working memory load would divert some of those resources. If nicotine enhances concentration (attention-to-task), then a concurrent working memory load should not influence nicotine-induced improvements in ProM performance. The study reported below examines the effects of nicotine, administered via nasal spray, on ProM performance. The ProM task is presented with and without an additional working memory load, to habitual nicotine users (smokers) and naïve users (nonsmokers).

\section{MATERIALS AND METHODS}

\section{Volunteers}

In all, 32 habitual smokers (5-15 cigarettes/day) and 33 'never-smoked' volunteers were recruited. Smokers (22 females, 10 males) had a mean age of 22.8 years (range 18-35 years), and nonsmokers ( 28 females, five males) had a mean age of 21.03 years (range 18-34 years). Smokers had a mean nicotine dependency score (Fagerström Tobacco Questionnaire; Fagerström, 1978) of 4.1 (range 1-9). All smoker volunteers were required to abstain from smoking for $2 \mathrm{~h}$ before the test session and compliance was monitored with a $\mathrm{CO}$ smokerlyser measure at arrival in the laboratory. Two participants registered excessively high
CO scores on arrival at the laboratory, and were excluded from subsequent analyses. Mean CO score for the remaining 30 smokers were $6.9 \mathrm{ppm}$ (range 1-17 ppm).

All participants volunteered under a written informed consent procedure, and were reimbursed for their participation. Sussex University School of Life Sciences Ethics Committee approved the studies.

\section{Materials}

The task comprised a computerized LDT with an embedded ProM task (adapted from Brandimonte et al, 2001). In the LDT task, each volunteer completed 192 trials comprising $50 \%$ word trials, $25 \%$ legal nonwords (ie pronounceable letter strings), and $25 \%$ illegal nonwords (ie unpronounceable letter strings). Each stimulus appeared four times across the 192 trials, in a computer-generated random sequence. Each stimulus was presented on the center of the screen in white upper-case letters on a black background, following the offset of a focal asterisk. The stimuli remained on screen for $500 \mathrm{~ms}$ and the volunteer was required to press one of two designated buttons: 'yes' for a word and 'no' for a nonword.

The ProM task was adapted from Kliegel et al (2004). Two 'target' letters ( $\mathrm{P}$ and $\mathrm{Q}$ ) were identified, each of which would appear, embedded within the LDT stimuli, four times within the entire sequence (in common with all other word and nonword stimuli). The stimuli containing these target letters were novel to each block, since repetition would allow the task to become a 'whole word' process on the second occasion, thus defeating the purpose of the manipulation. Volunteers were instructed that if they saw either of the target letters during the presentation, they should press the space bar to indicate that a target item had occurred (instead of making an LDT response for that stimulus).

In addition, for half of the volunteers, an auditory working memory task was incorporated. A random sequence of single digits (1-9) was recorded at a presentation rate of one digit every $2 \mathrm{~s}$, and this sequence contained a total of 30 instances of the digit ' 9 '. Volunteers in the memory load condition were instructed to monitor the auditory stream of digits occurring simultaneously with the LDT and ProM tasks, and to press the center button of the button box (positioned between the assigned word/ nonword response buttons) whenever a number 9 occurred in the auditory sequence. They were asked to respond to the digit before making the LDT response. The LDT responses occurring concurrently with the probe digit were discarded from the data set. No probe items occurred concurrently with the ProM target stimuli.

A practice set of 24 items, with the same proportion of words, legal and illegal nonwords, was devised. No ProM target items appeared within the practice set.

\section{Design}

The study was a double-blind placebo-controlled study. Nicotine nasal sprays and matched placebo sprays were provided by Pfizer Consumer Healthcare, Helsingborg, Sweden. The sprays were coded by an independent party. Half of the smokers and half of the nonsmokers received 
nicotine-containing sprays ( $n=16$ /group); neither the volunteer nor the experimenter were aware of the condition to which the volunteer was assigned. Half of the volunteers in each of these four groups completed the LDT/ProM task without the working memory load, and half completed it with the working memory task ( $n=8$ /group). This produced a 2 (smoker/nonsmoker $) \times 2$ (nicotine/placebo $) \times 2$ (WM load/no load) design against which to examine performance on the ProM task, LDT accuracy and reaction time, and working memory task accuracy.

\section{Procedure}

All volunteers visited the laboratory on the day before the test session, to familiarize themselves both with the technique for using the nasal spray correctly and with the sensory experience. Smoker volunteers were instructed to abstain from smoking for the 2 -h period prior to the test session (avoiding overnight nicotine deprivation and ensuring minimal deprivation). (Pilot data on an independent sample $(N=10)$ of $2-3 \mathrm{~h}$ abstinent moderate smokers indicated no significant change in QSU rating of craving or negative mood over time (means for QSU factor 1: 5.9, 5.9, $p>0.8$; QSU factor 2: 2.9, 3.9, $p>0.1$; QSU total: 4.4, 4.9, $p>0.2$.)

The test session began with an abstinence compliance check (smokers only) (see volunteer section). All volunteers then received instructions for the LDT/ProM task. They were instructed to complete the LDT task as quickly and accurately as possible, but to withhold the LDT response whenever a designated 'target' item occurred, and to press the space bar to indicate that the target item had been noted. From this point, the importance of the ProM task was not restated, and the practice set did not contain ProM exemplars. Volunteers in the 'WM load' condition were then given instructions for the ongoing auditory monitoring task. All volunteers then self-administered a single dose of nasal spray to each nostril (delivering approx. $1 \mathrm{mg}$ nicotine for the active group). Following an interval of $6 \mathrm{~min}$ (filled with administrative details and conversation), all volunteers completed the LDT/ProM task with or without the WM load.

\section{Data Compilation and Analysis}

From the raw data, each volunteer contributed the following measures: number of ProM targets noted during the LDT task (maximum $=8$ ); percentage correct of LDT trials (pressing 'yes' button to words, 'no' button to nonwords); median RTs to correct LDT trials; and RVIP performance measures (number of correctly recognized targets, mean RT to correct targets, number of incorrect button presses). In addition, volunteers in the WM load condition were scored for the number of correctly monitored target digits (maximum 30) and RTs to targets. Data was analyzed using ANOVA, and interactions explored with $t$-tests.

\section{RESULTS}

\section{LDT Performance}

Error rates were low $(<4.0 \%)$ and were unaffected by any of the manipulations. Reaction times to complete the LDT were analyzed using a 3 (word type: word, legal nonword, illegal nonword) $\times 2$ (group: smoker, nonsmoker) $\times 2$ (load: WM task, no task) $\times 2$ (condition: placebo nasal spray, nicotine nasal spray) mixed ANOVA, with the last three factors between subjects. In common with all LDT studies, words were responded to more quickly than nonwords $(\mathrm{F}(2,110)=29.1, p<0.001)$, but there were no interactions between word type and other factors (group, condition or load). Nonsmokers were faster overall in their LDT response times than were smokers $(\mathrm{F}(1,55)=3.95, p=0.052)$, but this effect was qualified by an interaction with WM load $(F(1,55)=6.18, p<0.02)$. Smokers were nonsignificantly faster in the no-load condition (mean RTs 579 and $610 \mathrm{~ms}$, respectively), but significantly slower when a WM load was imposed (mean RTs 980 and $704 \mathrm{~ms}$, respectively; $\mathrm{t}(29)=2.68, p<0.012$, two-tailed).

\section{Working Memory Task}

Volunteers asked to concurrently monitor the occurrence of a target digit in an auditory stream of random numbers performed this task extraordinarily well. On average, volunteers correctly identified 26.7 of the 30 possible targets, and this level of performance was independent of all other manipulations. There were almost no inappropriate responses (pressing the button to a digit other than the target ' 9 ') on this task. Reaction times to respond to the targets was also analyzed; there were no differential effects of group (smoker/nonsmoker) or of condition (nicotine/ placebo nasal spray).

\section{ProM Performance}

The number of correctly identified ProM targets ( $\max 8$ ) was analyzed using a 2 (group: smoker, nonsmoker) $\times 2$ (load: WM task, no task) $\times 2$ (condition: placebo nasal spray, nicotine nasal spray) between-subjects ANOVA. Although initial ProM task instructions asked participants to respond to the ProM target before making the LDT response, in practice volunteers often made the LDT response before registering the ProM target. In this analysis, pressing the space bar in the presence of the target cue was scored as correct regardless of whether an LDT response was performed first. There were no main effects of group or of condition (F's $<1)$. There was a main effect of load, with better performance when there was no concurrent WM task $(\mathrm{F}(1,55)=4.46, p<0.04)$, but this was qualified by a significant interaction between load and condition $(\mathrm{F}(1,55)=5.94, p<0.02)$.

Nicotine produced a nonsignificant improvement in ProM performance in the no-load condition (overall means: 6.1 and 4.8, respectively; $\mathrm{t}(30)=1.53, p<0.1$, one-tailed), but poorer performance than placebo when a WM load was imposed (overall means: 3.4 and 5.0, respectively; $\mathrm{t}(29)=2.07, p<0.05$, two-tailed). A working memory load impaired performance in the nicotine condition $(\mathrm{t}(30)=3.6$, $p<0.001)$; it did not depress performance in the placebo condition $(\mathrm{t}(29)=0.22, p>0.8)$. Again, volunteers made almost no inappropriate responses (responding to words other than correct ProM targets). (In the above analysis, pressing the space bar in the presence of the target cue was scored as correct regardless of whether an LDT response 


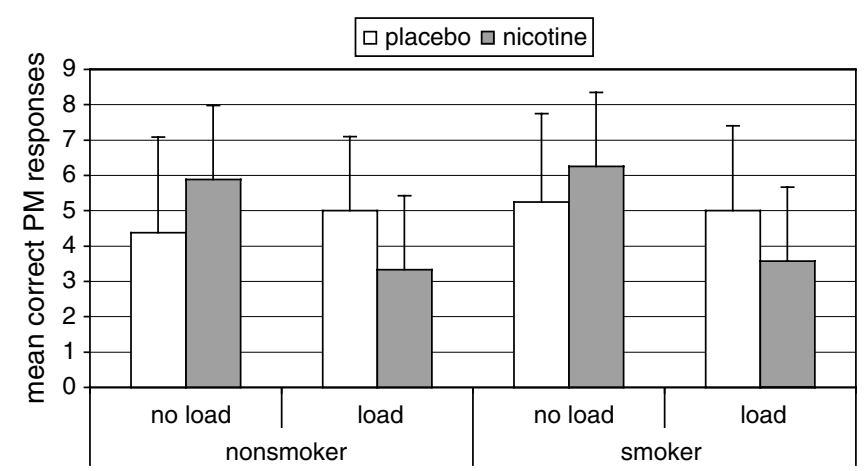

Figure I Mean ProM targets correctly identified as a function of group, nicotine condition, and WM load.

was performed first. In a stricter analysis, excluding any ProM responses that were preceded by an LDT response, the effect of nicotine in the no-load condition was more pronounced (means: 3.6 and 1.9, respectively, but nicotine made no reliable difference when a WM load was imposed (means: 2.0 and 2.7, respectively). This is consistent with the notion that these responses are based on strategic processing of the ProM cue, and that strategic ProM processing is neglected when a load is imposed.) The interaction was observed in both smokers and nonsmokers; Figure 1 provides a breakdown by group, demonstrating the consistency of the result.

\section{DISCUSSION}

In a double-blind placebo-controlled study, $1.0 \mathrm{mg}$ nicotine delivered via nasal spray significantly influenced the ability of both smokers and nonsmokers to complete accurately a ProM task. These effects were independent of previous exposure to nicotine and thus demonstrably independent of deprivation reinstatement, habitual usage, or smokingrelated confounds associated with the many studies that demonstrate positive effects of nicotine in smokers. In this instance, as in previous studies, the impact of nicotine is tightly dependent upon the cognitive demands of the situation.

This study explored prospective performance under two conditions: first, under standard conditions, with the ProM task embedded in an ongoing LDT, and secondly, when there was an additional concurrent working memory task. In the no-load condition, the trend for improved ProM performance replicated the Rusted et al (in press) result, in which nicotine delivered through smoking increased ProM accuracy on a similar paradigm. Significantly, the present study demonstrates equivalent effects of nicotine administered to smokers and to nonsmokers.

When a concurrent working memory task was introduced, however, ProM performance was impaired in the nicotine condition. An effect of WM load is consistent with the view that maintaining a ProM task engages resources; when other tasks compete for resources, the ProM task (and indeed the ongoing LDT task) is neglected. In this instance, diverting resources to the WM task did not improve performance on that task, possibly because performance was already at ceiling. That nicotine did not protect ProM accuracy under a WM load was somewhat surprising.

One explanation would be a simple arousal account of the behavioral effects of nicotine. Accordingly, nicotine heightened arousal in the no-load condition, and hence improved performance; but volunteers also experienced increases in arousal in response to the imposition of a WM load. The cumulative effects on arousal of drug + load pushed the volunteer beyond optimal arousal levels (an inverted U-curve, following the Yerkes-Dobson law), producing impaired performance with drug + load. This explanation accommodates the comparable ProM performance under placebo, independent of load (increased arousal counteracts the impairment associated with the WM load, pushing performance back up to baseline). Recent fMRI data reported by Kumari et al (2003) suggested a similar response in healthy, nonsmoking males who performed the $n$-back task following administration of either placebo or subcutaneous nicotine $(12 \mu \mathrm{g} / \mathrm{kg})$. Overall, nicotine improved accuracy of responding on the $n$-back task. This was associated with significantly decreased RTs in the high load (3-back) condition (volunteers rising to the challenging task), but nonsignificant increases in RTs on the low load (0-back) condition (RTs mapping lower basal arousal in this easy condition). The fMRI data did not support a strong relationship between nicotine effects and frontal activation, but rather between nicotine effects and distributed increases in neuronal activity in the anterior cingulate, superior parietal cortex, midbrain, and cerebellum. Together with the current data, the findings suggest that nicotine-related performance changes are most likely linked to nonspecific effects of nicotine on arousal, providing a rather small window of opportunity for enhancing higher-order cognitive processes. Sarter et al (2005), however, have suggested that discrepancies in the animal literature concerning frontal cholinergic activity changes in attention-demanding tasks may reflect a task-dependent mix of top-down cognitive mediation and bottom-up signal-driven activation of cholinergic pathways. This model offers an exciting prospect for exploration of similar inconsistencies in the human research literature.

In respect of current models of ProM, the present study indicates a key role for strategic processing in ProM. Nicotine improves ProM when the volunteer can allocate resources to the ProM task, but when resources are taxed, ProM processing may be neglected and nicotine-induced benefits lost. In short, nicotine administration does not enhance automatically any task that is ongoing. In this study, working memory demands reduced strategic processing of the intention, while in the Rusted et al study, subjective priorities determined strategic processing of the intention; in both cases, coadministration of nicotine failed to promote ProM performance.

In conclusion, the present study reports nicotine-related changes in ProM performance that are independent of smoking history. Equivalent effects are observed in nonsmokers and smokers. Nicotine improves ProM when the volunteer has no competing task demands, but the advantage is not maintained under cognitively demanding conditions. The results provide a clear link to the psychological models that incorporate ProM as a resourcedemanding process (eg McDaniel et al, 2004; Smith and 
Bayen, 2004). The demonstration of a neurobiological system that feeds this strategic engagement is a critical first step towards a comprehensive model of ProM. Currently, we are exploring the pharmacological specificity of these effects.

\section{ACKNOWLEDGEMENTS}

We thank Pfizer Consumer Healthcare, Helsingborg, Sweden, for supplying the nasal sprays. We also thank Sam Hutton and Dora Duka for discussion of earlier drafts of the paper.

\section{REFERENCES}

Brandimonte MA, Ferrante D, Feresin C, Delbello R (2001). Dissociating prospective memory from vigilance processes. Psicologica 22: 97-113.

Burgess PW, Scott SK, Frith CD (2003). The role of the rostral frontal cortex (area 10) in prospective memory: a lateral versus medial dissociation. Neuropsychologia 41: 906-918.

Burgess PW, Quayle A, Frith CD (2001). Brain regions involved in prospective memory as determined by positron emission tomography. Neuropsychologia 39: 545-555.

Cherry KE, LeCompte DC (1999). Age and individual differences influence prospective memory. Psychol Aging 14: 60-76.

Edginton T, Rusted JM (2003). Separate and combined effects of scopolamine and nicotine on retrieval-induced forgetting. Psychopharmacology 170: 351-357.

Einstein GO, McDaniel MA (1990). Normal ageing and prospective memory. J Exp Psychol: Learn Mem Cogn 16: 717-772.

Einstein GO, McDaniel MA (1996). Retrieval processes in prospective memory: theoretical approaches and some new empirical findings. In: Brandimonte MA, Einstein GO, McDaniel MA (eds). Prospective Memory: Theory and Applications. Erlbaum: Hillsdale, NJ. pp 115-141.

Einstein GO, McDaniel MA, Thomas R, Mayfield S, Shank H, Morrisette $\mathrm{N}$ et al (2005). Multiple processes in prospective memory retrieval: factors determining monitoring versus spontaneous retrieval. J Exp Psychol-Gen 134: 327-342.

Ellis J, Kvavilashvili L (2000). Prospective memory in 2000: past, present, and future directions. Appl Cogn Psych 14: S1-S9.

Ernst M, Matochik JA, Heishman SJ, Van Horn JD, Jons PH, Henningfield JE et al (2001). Effect of nicotine on brain activation during performance of a working memory task. Proc Natl Acad Sci USA 98: 4728-4733.

Fagerström KO (1978). Measuring degree of physical dependence to tobacco smoking with reference to individualization of treatment. Addict Behav 3: 235-241.

Huppert FA, Beardsall L (1993). Prospective memory impairment as an early indicator of dementia. J Clin Exp Neuropsychol 15: 805-821.

Kidder D, Park D, Hertzog C, Morrell RW (1997). Prospective memory and aging: the effects of working memory and prospective memory task load. Aging Neuropsychol Cogn 4: 93-112.

Kliegel M, Martin M, McDaniel MA, Einstein GO (2004). Importance effects on performance in event-based prospective memory tasks. Memory 12: 553-561.
Kliegel M, Martin M, Moor C (2003). Prospective memory and ageing: is task importance relevant? Int J Psychol 38: 207-214.

Kumari V, Gray JA, Ffytche DH, Mitterschiffthaler MT, Das M, Zachariah E et al (2003). Cognitive effects of nicotine in humans: an fMRI study. Neuroimage 19: 1002-1013.

Levin ED, Conners CK, Silva D, Hinton SC, Meck WH, March J et al (1998). Transdermal nicotine effects on attention. Psychopharmacology 140: 135-141.

Logie R, Maylor E, Della Sala S, Smith G (2004). Working memory in event- and time-based prospective memory tasks: effects of secondary demand and age. Eur J Cogn Psychol 16: 441-456.

Mancuso G, Warburton D, Melen M, Sherwood N, Tirelli E (1999). Selective effects of nicotine on attentional processes. Psychopharmacology 146: 199-204.

Mantyla T, Nilsson LG (1997). Remembering to remember in adulthood: a population-based study on aging and prospective memory. Aging Neuropsychol Cogn 4: 81-92.

Maylor EA (1996). Age-related impairment in an event-based prospective-memory task. Psychol Aging 11: 74-78.

McDaniel MA, Einstein GO (2000). Strategic and automatic processes in prospective memory retrieval: a multiprocess framework. Appl Cogn Psychol 14: 127-144.

McDaniel MA, Guynn MJ, Einstein GO, Breneiser J (2004). Cue-focused and reflexive-associative processes in prospective memory retrieval. J Exp Psychol: Learn Mem Cogn 30: 605-614.

Nowinski JL, Dismukes RK (2005). Effects of ongoing task context and target typicality on prospective memory performance: the importance of associative cueing. Memory 13: 649-657.

Okuda J, Fujii T, Yamadori A, Kawashima R, TsukiaraT, Fukatsu R et al (1998). Participation of the prefrontal cortex in prospective memory: evidence from a PET study in humans. Neurosci Lett 253: $127-130$.

Robbins TW (2002). The 5-choice serial reaction time task: behavioural pharmacology and functional neurochemistry. Psychopharmacology 163: 362-380.

Rusted JM, Graupner L, Tennant A, Warburton DM (1998). Effortful processing is a requirement for nicotine-induced improvements in memory. Psychopharmacology 138: 362-368.

Rusted JM, Trawley S, Kettle G, Walker H (in press). Nicotine improves memory for delayed intentions. Psychopharmacology.

Rycroft N, Rusted JM, Hutton SB (2005). Acute effects of nicotine on visual search tasks in young adult smokers. Psychopharmacology 181: 160-169.

Sarter M, Hasselmo ME, Bruno JP, Givens B (2005). Unraveling the attentional functions of cortical cholinergic inputs: interactions between signal-driven and cognitive modulation of signal detection. Brain Res Rev 48: 98-111.

Shallice T, Burgess P (1996). The domain of supervisory processes and temporal organisation of behaviour. Philos Trans $R$ Soc Lond 351: 1405-1412.

Smith RE, Bayen UJ (2004). A multinomial model of eventbasedprospectivememory. J Exp Psychol-Learn Mem Cogn 30: 756-777.

Trawley S, Rusted JM. Prospective memory benefits from contextual support: differential effects of working memory load. J Exp Psychol: Hum Learn Mem (under review).

Warburton DM, Skinner A, Martin CD (2001). Improved incidental memory with nicotine after semantic processing, but not after phonological processing. Psychopharmacology 153: 258-263. 